OAI-PMH: http://www.indteca.com/ojs/index.php/Revista Scientific/oai

Artículo Original / Original Article

\title{
Hermenéutica de la Flexibilidad Cognitiva de las Decisiones Estratégicas Empresariales desde la Transcomplejidad
}

\author{
Autora: María Auxiliadora Pérez \\ Universidad Centroccidental "Lisandro Alvarado", UCLA \\ maperez@ucla.edu.ve \\ Lara, Venezuela \\ http://orcid.org/0000-0002-3663-4895
}

Resumen

En el mundo empresarial de hoy, dinamizado por el ritmo exponencial de los cambios tecnológicos y una altísima competitividad, el ecosistema empresarial global, requiere que los directores ejecutivos que conforman equipos de alta dirección amplíen su percepción de este nuevo contexto y de las capacidades individuales que pueden aportar en el complejo mundo de las decisiones estratégicas. Adoptando la postura epistemológica de la transcomplejidad y enmarcada en el paradigma interpretativo, en esta investigación indago el papel que juega la flexibilidad cognitiva en las decisiones estratégicas empresariales, interpretando los hallazgos que emergieron del proceso intersubjetivo con los informantes clave. Por tanto, el enfoque metodológico utilizado es el cualitativo, bajo la corriente filosófica de la fenomenología apoyada en la hermenéutica. Como resultado se obtuvo que la flexibilidad cognitiva en las decisiones estratégicas empresariales se configura como la capacidad del alto ejecutivo para percibir el cambio, explorar nuevas ideas, adoptar nuevos enfoques, centrado en el conocimiento de sí mismo y su experiencia profesional, que le permite romper paradigmas y crear cosas, explorar nuevas ideas y asumir con audacia nuevos retos.

Palabras clave: actitud; análisis cualitativo; gestión; percepción. 


\title{
Hermeneutics of the Cognitive Flexibility of Business Strategic Decisions from Transcomplexity
}

\begin{abstract}
In the business world today, boosted by the exponential pace of technological changes and high competitiveness, the global business ecosystem requires managers and in particular members of senior management teams to broaden their perception of this new context and the individual capacities that can contribute in the complex world of strategic decisions. Adopting the epistemological posture of transcomplexity and framed in the interpretive paradigm, in this research I explore the role played by cognitive flexibility in strategic business decisions, interpreting the findings that emerged from the intersubjective process with key informants. Therefore, the methodological approach used is the qualitative, under the philosophical current of phenomenology supported by hermeneutics. The result was that the cognitive flexibility in strategic business decisions is configured as the ability of the top executive to perceive change, explore new ideas, adopt new approaches, focused on the knowledge of himself and his professional experience, allows breaking paradigms and create things, explore new ideas and boldly take on new challenges.
\end{abstract}

Keywords: attitude; qualitative analysis; management; perception. 


\section{Introducción}

En la década y media que ha transcurrido del siglo XXI, la sociedad se encuentra en una nueva etapa de desarrollo, donde la información y el conocimiento, la innovación tecnológica, la alta competitividad empresarial y el fenómeno de la globalización, entre otros, integran el escenario donde los altos directivos toman las decisiones estratégicas que orientan el éxito y sustentabilidad de sus respectivas organizaciones.

Es así como, tomar decisiones se constituye en una de las principales funciones del quehacer organizacional de todo gerente, donde cada decisión es importante en la medida que pueda generar valor para la organización y mejorar su desempeño.

Ahora bien, el proceso de toma de decisiones estratégicas como fenómeno exhibe características complejas, puesto que conecta relaciones, intereses, contradicciones, riesgos y beneficios. Superada la tesis del actuar racional como patrón universal del comportamiento de los agentes de la economía social, entre los cuales los gerentes juegan un papel esencial, las decisiones se encuentran insertas en la dinámica social organizacional, cuyo conocimiento parece necesario a la hora de comprender los cimientos y pilares que guían el proceso de decisiones estratégicas de los directores ejecutivos.

Al mismo tiempo, Maldonado (2008): señala que con el acercamiento entre ciencias duras y sociales, "no solamente las ciencias de la complejidad han ganado extensión y fortaleza sino, lo que es aún más significativo, la comprensión de los procesos, estructuras y dinámicas de los sistemas sociales humanos se han enriquecido enormemente y ganado universalidad" (pág. 154).

Desde el punto de vista gerencial, el sustento del éxito empresarial se encuentra directamente vinculado a las decisiones que toman sus directores ejecutivos en diversos ámbitos, desde el financiero, las relaciones públicas, la gestión del talento humano, el mercadeo, entre otras áreas, hasta la estrategia 
y la planificación. Como fenómeno complejo, planteo que entre las habilidades directivas a integrar en el proceso decisional se encuentra la flexibilidad cognitiva, como competencia primordial para el desarrollo del potencial de los directores ejecutivos en materia de toma de decisiones.

Hechas las consideraciones anteriores y señalada por Gray (2016): entre "las diez competencias necesarias para prosperar en la Cuarta Revolución Industrial" (párr. 6); en el Foro Mundial de Davos, en esta investigación indago el papel que juega la flexibilidad cognitiva en las decisiones estratégicas empresariales desde una perspectiva transcompleja.

De acuerdo con lo planteado por el autor, la flexibilidad cognitiva implica que la persona experimente una especie de modificación estructural cognitiva al relacionar pensamientos e ideas, de modo no arbitrario y sustancial, trasladando a nuevos contextos el conocimiento adquirido.

En lo que se refiere a gestión empresarial, el Portal de la Escuela de Negocios (EAE, 2015a): considera que la flexibilidad cognitiva es "la capacidad de moldear, adaptar y aplicar el conocimiento y la experiencia adquiridos a distintas áreas, actividades y procesos - en nuestro caso (y lógicamente) relacionados con la gestión empresarial" (párr. 2).

De igual forma plantea (EAE, 2015b): la flexibilidad cognitiva correctamente administrada, permite afrontar de "modo óptimo, escenarios cambiantes, adaptar actividades, funciones y comportamientos a los requerimientos específicos de cada nuevo contexto... asumir tareas y responsabilidades múltiples, complejas y eventualmente descontextualizadas" (párr. 3).

Por otra parte, el enfoque de la neurociencia cognitiva planteado por Matute (2012): establece que la flexibilidad cognitiva "se refiere a la posibilidad de ajustar el comportamiento ante el cambio de exigencias o contingencias ambientales" (pág. 102).

En este mismo orden de ideas, Braidot (2016): define la flexibilidad 
cognitiva como "la habilidad para analizar varias alternativas en forma paralela o simultánea y realizar los ajustes que se necesitan cuando cambia el contexto. Dado que requiere de la imaginación, esta habilidad está relacionada con la creatividad" (párr. 5).

Señala el autor que resultados individuales, como el éxito en el trabajo, el desempeño sobresaliente en los estudios académicos tanto a nivel de escuela como universitario, en la vida social y afectiva, reflejan la buena observancia de las funciones ejecutivas, que dependen, entre otras, de la flexibilidad cognitiva.

Es este mismo sentido, CogniFit (2018a), define la flexibilidad cognitiva como:

la capacidad que tiene nuestro cerebro para adaptar nuestra conducta y pensamiento a situaciones novedosas, cambiantes 0 inesperadas. En otras palabras, es la facultad de darnos cuenta de que lo que estamos haciendo no funciona, o ha dejado de funcionar $y$, por tanto, debemos reajustar nuestra conducta, pensamiento y opiniones para adaptarnos al entorno y a las nuevas situaciones (párr. 1).

A propósito del impacto de la flexibilidad cognitiva sobre el grado de racionalidad del proceso de toma de decisiones estratégicas, en las conclusiones del trabajo de Pedraja, Rodríguez y Rodríguez (2008), puede apreciarse que esta capacidad resulta efectivamente representativa en la racionalidad del decisor por lo que su propuesta es favorecer esta competencia en los gerentes de alto nivel, estimulando: "la discusión de muchas ideas diferentes, el análisis de múltiples perspectivas o enfoques diferentes, las diferencias de opinión entre los participantes y la incorporación de nueva información en forma recurrente" (pág. 152).

Asimismo, entre los hallazgos de la investigación de Rodríguez y Pedraja (2009): se tiene que "la flexibilidad cognitiva impacta positiva y significativamente sobre la calidad de las decisiones estratégicas en las 
empresas públicas" (pág. 43); constituyendo de esta forma, un componente estructural de la racionalidad del proceso decisional estratégico.

Vinculada directamente con la capacidad de cambio, la flexibilidad cognitiva conforma su base fundamental. A la vez, CogniFit (2018b): refiere la diferencia entre el cambio mental y la adaptación al cambio, señalando que "la flexibilidad cognitiva se refiere a la capacidad de adaptarnos al cambio, el cambio mental se refiere al proceso por el cual nos adaptamos a dicho cambio" (párr. 2).

Adicionalmente, desde la perspectiva empresarial resulta oportuno destacar el papel prominente de la flexibilidad cognitiva en la resolución de problemas complejos, dado que esta capacidad permite a los directores ejecutivos escoger una estrategia de un conjunto de posibles opciones para dar respuesta a las distintas situaciones que se presentan, realizando el reajuste de pensamientos y acciones en función de la información y las exigencias captadas del ambiente, haciéndolo de forma flexible.

Así, el desarrollo de la flexibilidad cognitiva nos otorga la posibilidad de ajustarnos ágilmente a los cambios 0 acontecimientos, manejar adecuadamente los imprevistos que ocurren durante la resolución de problemas, generando múltiples alternativas de solución.

Como decisores estratégicos, considerar el punto de vista de los miembros del equipo, admitir el pensamiento divergente del otro, acoger otras formas de pensar incluidas creencias y valores, es una expresión sustancial de la praxis gerencial resultante de incorporar la flexibilidad cognitiva en el proceso de tomar decisiones.

En resumen, desarrollar la capacidad de percibir la realidad multidimensional del mundo gerencial en un momento específico desde diferentes perspectivas decidiendo sobre asuntos complejos, requiere muy particularmente, reajustarse rápidamente a los cambios, muchas veces corregir el rumbo, tolerar los errores, es decir, ser flexible cognitivamente. 


\section{Aspectos teóricos}

\subsection{Teoría de la Complejidad}

Desde la concepción de Morin (1998): "la complejidad es, efectivamente, el tejido de eventos, acciones, interacciones, retroacciones, determinaciones, azares, que constituyen nuestro mundo fenoménico" (pág. 32). Igualmente, en el contexto actual Morin (2014a): plantea que, "la complejidad también es un modo de conocimiento cuando integramos ciertos principios: el principio de retroactividad, de conectividad, en un principio dialógico. Es una forma de pensar" (pág. 19).

Asimismo, desde el punto de vista sistémico para el estudio y comprensión de fenómenos complejos, Morin (2014b), expresa:

En mi opinión, el concepto de sistemas nos proporciona la información esencial necesaria para adquiriendo conocimiento de fenómenos complejos, en el sentido de que cuando comenzamos a mirar la ciencia clásica desde una perspectiva crítica; vemos que los "objetos" estudiados por la ciencia, y tratados por la ciencia simplemente como objetos de hecho son todos los sistemas (pág. 15).

Adicionalmente señala Martínez (2011): "nuestras realidades cambian según nos encontremos en un nivel de diferente organización o campo" (pág. 8); de manera tal que, para ser aprehendida y expresada, la realidad demanda de un pensamiento complejo.

En particular, la cosmovisión de la complejidad que asume esta investigación, es una actitud de diálogo delante de los fenómenos aceptando lo paradójico, la incertidumbre y lo que no acabado como propiedad de los fenómenos.

Sobre la aproximación al quehacer gerencial desde el enfoque complejo y transcomplejo, Figueredo (2017), realiza el siguiente planteamiento:

El fundamento teórico y praxeológico de la gerencia, está impregnado de múltiples interacciones, relacionalidades y enfoques disciplinares. No hay un conocimiento específico que 
brinde o aporte todos los atributos que exhibe la gerencia y aprehende y aplica el gerente. La gerencia tanto en su discurso teorizante como en su práctica cotidiana, es una especie de crisol donde se forja un conocimiento puesto al servicio del colectivo y a la permanente búsqueda del bienestar humano como propósitos cimeros. La visión transcompleja de la gerencia se orienta entonces a una permanente observación y comprensión de las rupturas paradigmáticas y a las emergencias epistémicas (pág. 330).

Es así como el contexto teórico investigativo está conformado por los aportes de la Cibernética Social o de segundo orden, que permitieron superar la noción de la duplicidad de la realidad externa en la mente del sujeto observador, presentando la circularidad, la retroalimentación, la regulación, la información y la meta y además del orden, el azar y el ruido, entre otros, la Teoría de los Sistemas Sociales para la comprensión y explicación de la complejidad a través de la construcción de una red sistémica de la realidad, donde se procesan de forma selectiva situaciones, elementos y alternativas que permiten transformar la complejidad que caracteriza una diversidad de eventos humanos, hasta la teoría del pensamiento complejo, cuando Morín y Le Moigne (2006): plantean que "todo sistema, cualquiera que sea, es (o puede entenderse) complejo en su naturaleza misma" (pág. 23).

En particular, tomando como referencia el enfoque de transcomplejidad de Morín, planteo que la realidad que percibimos es el resultado de una permanente construcción de eventos entrelazados, acciones e interacciones que determinan las decisiones y acciones que tomamos y que constituye el desempeño que demuestra todo director ejecutivo sobre el rumbo y destino de la organización que dirige.

\subsection{Teoría de la Flexibilidad Cognitiva}

Centrada en la naturaleza del aprendizaje en dominios no estructurados y complejos, la Teoría de Flexibilidad Cognitiva referida por Spiro, Feltovich, 
Jacobson, y Coulson (1999a): se enfoca en la "adquisición del conocimiento, como el reensamblaje flexible del conocimiento preexistente para adaptarse a una nueva situación de acuerdo con nuevas necesidades" (pág. 87). Los autores plantean que no solo recuperamos paquetes de conocimiento antiguo, sino que los ensamblamos para formar nuevas realidades relacionadas.

Adicionalmente, Spiro, Feltovich, Jacobson, y Coulson (1999b), afirman que la flexibilidad cognitiva es la capacidad de:

reestructurar espontáneamente nuestro conocimiento, de muchas maneras, en respuesta adaptativa a demandas situacionales radicalmente cambiantes, en función a la forma en que se representa el conocimiento (por ejemplo, a lo largo de múltiples dimensiones conceptuales bastante simples) y los procesos que operan en esas representaciones mentales (por ejemplo, procesos de ensamblaje de esquemas en lugar de recuperación de esquemas intactos), (pág. 165).

En el entorno de las decisiones ejecutivas empresariales, la teoría de la flexibilidad cognitiva refleja la complejidad que normalmente enfrentan los directores ejecutivos; el contenido y las actividades relacionadas al proceso decisional, frente a la no linealidad de las decisiones y a los diferentes dominios del quehacer gerencial.

Respecto a la Teoría Cognitiva del Aprendizaje, ésta aborda al cerebro como una red en permanente construcción, basada en un enfoque ampliado de la multiplicidad de procesos cognitivos que intervienen para que el aprendizaje ocurra en el individuo. Según Peña, Cañoto, y Santalla (2006): la Teoría Cognitiva del Aprendizaje define la dimensión cognoscitiva de la conducta, "consideraba que los seres humanos no respondían simplemente a estímulos, sino que su comportamiento era guiado por creencias y actitudes y que se esforzaban por alcanzar metas" (pág. 172).

En el contexto de las decisiones estratégica, esta teoría aporta un conjunto de los elementos que permiten caracterizar al proceso decisional 
como fenómeno emergente transcomplejo.

\section{Metodología}

La reflexión sobre el proceso de crear conocimiento, supone un análisis crítico y conlleva la adopción de un paradigma epistémico. En particular, el paradigma que asumo en esta investigación es el interpretativo, centrado en la comprensión de la realidad social desde los significados de los sujetos implicados, estudiando las creencias, intenciones, motivaciones y otras cualidades de hechos sociales no observables directamente, ni susceptibles de experimentación, de la cual, producto de la interacción dialéctica entre el sujeto cognoscente y objeto cognoscible, emerge el conocimiento.

A la vez, el abordaje de la investigación lo realice mediante técnicas cualitativas. Se realizaron entrevistas a profundidad a tres (03) informantes clave que se desempeñan como directores ejecutivos de empresas del sector alta tecnología. Una vez grabadas y transcritas las entrevistas, analicé cada uno de los actores sociales y realicé la comparación entre sí de los testimonios provistos.

En lo que se refiere a la unidad de análisis utilizada, la misma fue una decisión estratégica tomada recientemente con el equipo de alta dirección en la organización donde prestan sus servicios, que comprometió una considerable cantidad de recursos económicos, tecnológicos y humanos, que abarcó un alto número de funciones organizacionales, con alto impacto tanto interno y como externo y cuyos efectos se estiman a largo plazo.

Resulta oportuno señalar que las categorías encontradas están combinadas o sobrepuestas. De igual forma se utilizó la triangulación metodológica de datos, investigadores y teorías, como herramienta para manejar la influencia de la subjetividad de la investigadora en la interpretación de los textos y de los entrevistados. 


\section{Hallazgos de la investigación}

A partir del testimonio de los informantes clave y utilizando la herramienta de software en línea y de computación en la nube para pensamiento visual denominada Inspiration Software, organicé la información para posteriormente modelarla mediante redes semánticas para cada uno de los ejes de estudio emergentes.

Primeramente, en términos generales ante situación que requiera tomar una decisión estratégica de gran impacto para la organización que dirige. mi primer informante clave señala que su proceso decisional se encuentra en permanente transformación (la toma de decisiones está marcada por la necesidad de cambio); que surge como respuesta a la exploración permanente del mercado donde se desenvuelve (teniendo en consideración estudios serios del contexto mundial, las tendencias tecnológicas del futuro y siguiendo los lineamientos de transformación los grandes líderes en el mercado); que en su caso particular se materializa en la concreción de un nuevo modelo de negocios (de un modelo de negocio basado en venta y soporte de infraestructura tecnológica... a una empresa centrada en un modelo de negocio de servicio); y demanda explorar nuevas ideas (La decisión es entonces, que la organización se transforme en una empresa que ofrezca Servicios en Cloud bajo las premisas del CAMSS).

Asimismo, señala, las decisiones estratégicas requieren de la adopción de nuevos enfoques, (apuntan hacia un mundo más inteligente); e implica superar la resistencia al cambio en un mundo caracterizado por un ritmo exponencial de producción de conocimiento en el ámbito empresarial (soportar los constantes cambios que exige el mercado. Siempre hay temor hacia la transformación, pero cuando la transformación se trata de un asunto de vida o muerte entonces aceptas la transformación como un camino que exige innovación y nuevas potencialidades a explorar y conquistar).

En este mismo sentido, interpreto de mi informante clave, la necesidad 
de reformular nuestro propio conocimiento como factor primordial de las decisiones estratégicas empresariales (siempre es al principio en donde surgen las dudas sobre el éxito de la decisión y sobre el cambio que debes emprender. Una vez que analizas los escenarios y entiendes que es necesario la transformación o más radicalmente la necesidad de mutación, aceptas el reto y comienza el trabajo de preparación para afrontar el reto). Figura nro. 1 muestra un modelo de las apreciaciones e interpretación de los aportes del informante clave 1.

Figura nro. 1. Modelo, flexibilidad cognitiva, informante clave 1.

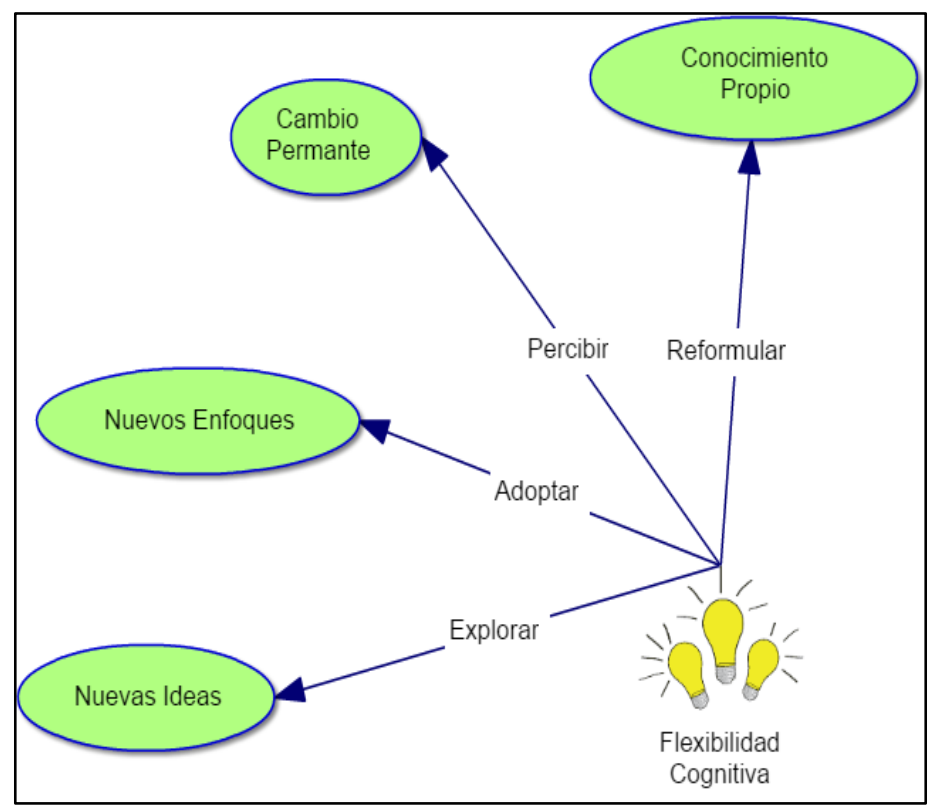

Fuente: La Autora (2018).

Ante la situación planteada, mi segundo informante clave plantea que la base de las decisiones estratégicas que toma es su experiencia profesional (mi experiencia, mi capacidad profesional); centrado en el conocimiento de sí mismo (quería producir un producto de exportación de clase mundial y les pareció bastante interesante y creo que el producto, creo no, estoy ya montado en la creación del nuevo producto...); y la transferencia de conocimiento 
(Termino esta pregunta, recordando a Edward Deming, que es conocido como el padre de la calidad en Japón. Deming cuenta una de sus historias que cuando llego a Japón... para poder llevar adelante este país (les estaba hablando a los japoneses), les indicó que la manera de salir adelante era produciendo productos para la exportación y para producir esos productos y poderlos exportar tenían que mejorar significativamente la calidad de estos, Bueno más o menos en ese orden de ideas, es donde yo estoy trabajando. He mejorado significativamente la calidad de mi producto. Le he colocado toda la tecnología, no toda la tecnología, pero si buena parte de la tecnología que yo conozco y me he asesorado), para explorar nuevas ideas (Me convencí de que cualquier producto que hoy en día uno tenga que mercadear en Venezuela tiene que ser con nivel internacional, tiene que ser para exportación) y asumir con audacia nuevos retos (mi osadía para tomar las cosas, para tomar los riesgos. Realmente he tenido la osadía siempre en mi vida de ir a grandes organizaciones a ofrecer mis servicios y de asumir retos importantes). La Figura nro. 2, muestra la red semántica resultante de la interpretación de la praxis de la flexibilidad cognitiva suministrada por mi actor social.

Figura nro. 2. Modelo, flexibilidad cognitiva, informante clave 2.

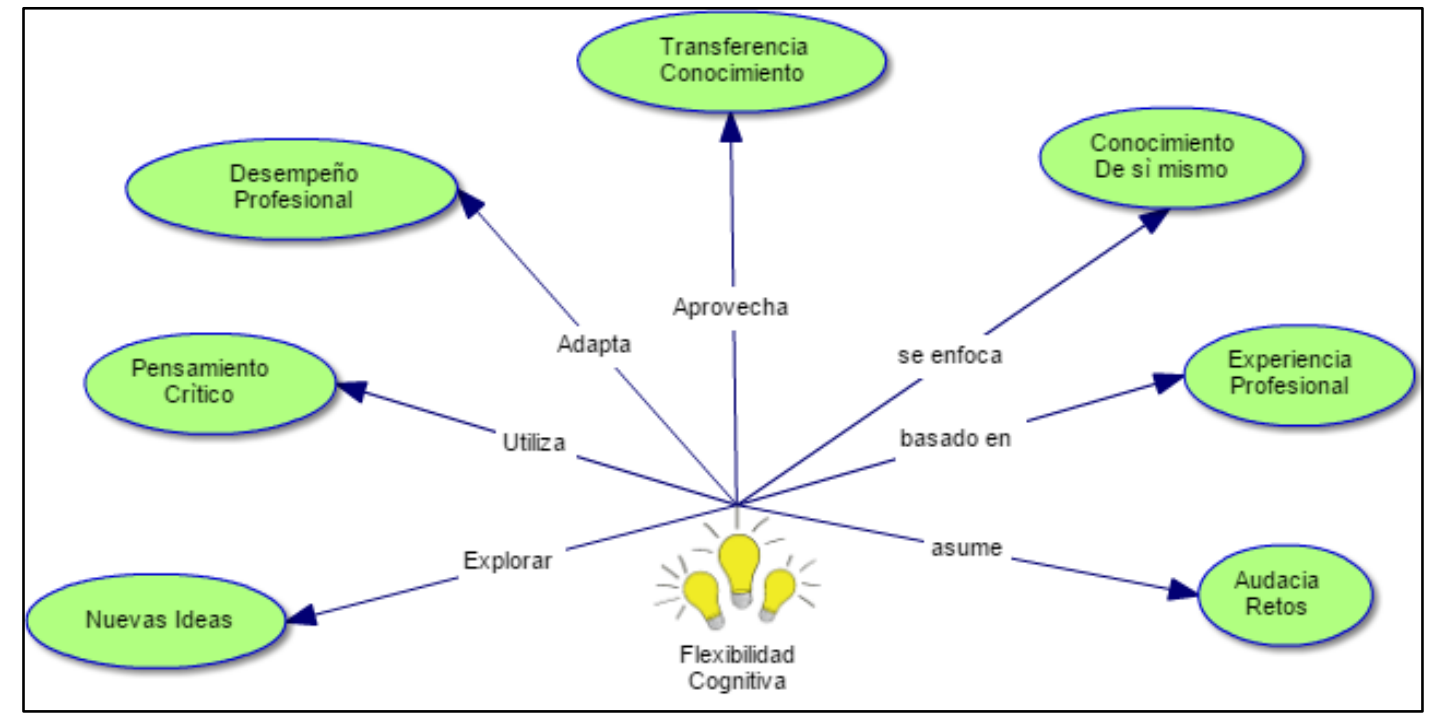

Fuente: La Autora (2018). 
Por otra parte, el análisis del testimonio de la flexibilidad cognitiva facilitado por mi tercer informante clave en las decisiones estratégicas (Figura nro. 3), la manifiesta como capacidad que le permite cambiar de estrategia como respuesta a la percepción de cambios en el entorno (cambiamos la forma en que mercadeamos nuestros productos); explorando nuevas ideas (cambiamos la figura de vender el producto y los términos de renovación del servicio de soporte para el año que viene y a veces nos decían que no, lo cambiamos a alquilar, entonces hablamos de software de alquiler); manejando múltiples alternativas (vimos la oportunidad de que los clientes querían mejores precios, teníamos el conocimiento de convertir cualquier tipo de actividad en servicio, nos enfocamos en esa filosofía y gustó).

Figura nro. 3. Modelo, flexibilidad cognitiva, informante clave 3.

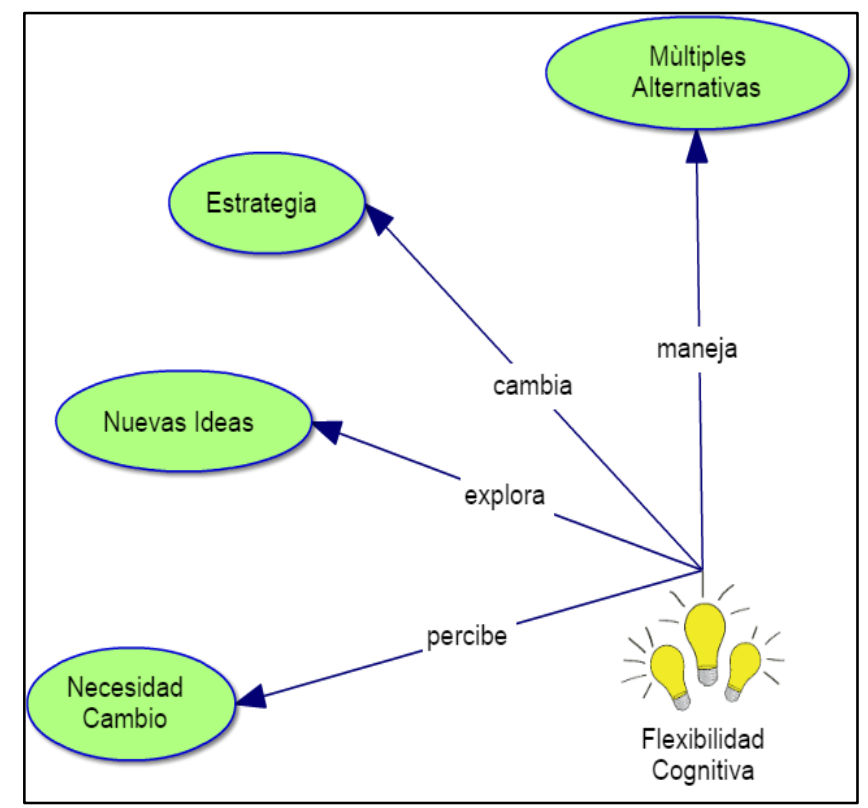

Fuente: La Autora (2018).

\section{Reflexiones Finales}

De acuerdo con los razonamientos que he venido realizando, la flexibilidad cognitiva en las decisiones ejecutivas empresariales se concibe 
como la capacidad del alto ejecutivo para percibir el cambio permanente del entorno empresarial, explorar nuevas ideas y adoptar nuevos enfoques, centrado en el conocimiento de sí mismo y su experiencia profesional, lo que le permite romper paradigmas y crear cosas, explorar nuevas ideas y asumir con audacia nuevos retos.

El papel esencial de la flexibilidad cognitiva, es uno de los aspectos principales del proceso de toma de decisiones estratégicas gerenciales, ya que nos ofrece la posibilidad de variar de estrategia en un determinado curso de acción cuando los resultados obtenidos se desvían de nuestro objetivo, captando diferentes perspectivas de una misma realidad y descubrir múltiples matices de un mismo problema. Una mente flexible nos permite percibir la realidad en términos de oportunidades y responder creativamente.

Dado que el conocimiento es una construcción permanente del cerebro y de ningún modo, un modelo terminado, cuya complejidad asociada incluye la consideración de numerosos elementos, en particular, la flexibilidad cognitiva; y el proceso de toma de decisiones estratégicas básicamente se fundamenta en el conocimiento, éste entretejido de interconexiones relacionales es lo que permite a los altos ejecutivos adaptarse al entorno empresarial y tomar mejores y eficientes decisiones.

En este sentido, la postura que asumo es por tanto que, a partir de nuestra percepción del entorno y utilizando nuestra capacidad de flexibilidad cognitiva, emergen múltiples posibilidades de reentender y resignificar la realidad, lo que influye significativamente en la forma en que los miembros de equipos de alta dirección toman decisiones estratégicas.

\section{Referencias}

Braidot, N. (2016). El Cerebro Ejecutivo. Buenos Aires, Argentina: Braidot. Recuperado de: https://braidot.com/el-cerebro-ejecutivo/

CogniFit (2018a,b). Flexibilidad Cognitiva. Israel: CogniFit. Recuperado de: 
https://www.cognifit.com/es/flexibilidad-cognitiva

EAE (2015a,b). La flexibilidad cognitiva, un valor en alza en la empresa.

Retos en Supply Chain. Barcelona, España: Blog de EAE Business

School. Recuperado de: https://retos-operaciones-logistica.eae.es/la-

flexibilidad-cognitiva-un-valor-en-alza-en-la-empresa/

Figueredo, C. (2017). La Gerencia Avanzada en el Proceso de Investigación Científica Universitaria. Revista Scientific, 2(6), 322-

336. Recuperado de: https://doi.org/10.29394/scientific.issn.25422987.2017.2.6.17.322-336

Gray, A. (2016). The 10 skills you need to thrive in the Fourth Industrial Revolution. New York and San Francisco, EE. UU.: World Economic Forum LLC. Recuperado de:

https://www.weforum.org/agenda/2016/01/the-10-skills-you-need-tothrive-in-the-fourth-industrial-revolution/

Maldonado, C. (2008). Complejidad y ciencias sociales desde el aporte de las matemáticas cualitativas. Cinta de Moebio, (33), 153-170, ISSN: 0717-554X. Recuperado de:

https://www.moebio.uchile.cl/33/maldonado.html

Martínez, M. (2011). El Paradigma Sistémico, la Complejidad y la Transdisciplinariedad como Bases Epistémicas de la Investigación Cualitativa. Revista Electrónica de Humanidades, Educación y Comunicación Social, REDHECS, 11(6), 6-27, ISSN-e: 1856-9331. Recuperado de:

http://prof.usb.ve/miguelm/El\%20paradigma\%20sistemico\%20\%20complej\%20y\%20transdisc.html

Matute, E. (2012). Tendencias actuales de las neurociencias cognitivas. 2da. Edición, ISBN: 978-607-448-273-7. México, D.F.: Editorial S.A. de C.V. Recuperado de:

https://books.google.co.ve/books?id=3bPKCQAAQBAJ\&lpg=PA108\&ot 


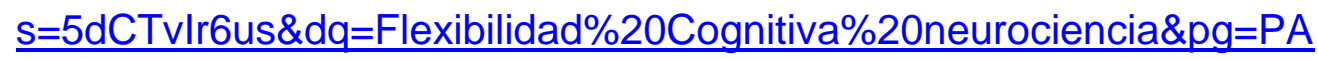
$108 \# v=$ =nepage \& $q=$ =Flexibilidad\%20Cognitiva\%20neurociencia\&f=false Morin, E. (1998). Introducción al pensamiento complejo. Barcelona España: Editorial Gedisa.

Morin, E. (2014a,b). Complex Thinking for a Complex World - About Reductionism, Disjunction and Systemism. Systema: connecting matter, life, culture and technology, 2(1), 14-22, ISSN: 2305-6991. Recuperado de: http://www.systema-journal.org/article/view/257

Morín, E., \& Le Moigne, J. (2006). Inteligencia de la Complejidad Epistemología y Pragmática. París, Francia: Ediciones de l'aube.

Pedraja, L., Rodríguez, E., \& Rodríguez, J. (2008). Determinantes del Éxito en la formulación de decisiones estratégicas en instituciones universitarias. Calidad en la Educación, (29), 138-158. Recuperado de: https://doi.org/10.31619/caledu.n29.191

Peña, G., Cañoto, Y., \& Santalla, Z. (2006). Una Introducción a la piscología. Caracas, Venezuela: Universidad Católica Andrés Bello.

Rodríguez, E., \& Pedraja, L. (2009). Análisis del impacto del proceso de toma de decisiones estratégicas sobre la eficacia de las organizaciones públicas. Innovar, 19(35), 33-46, ISSN-e: 2248-6968. Recuperado de:

https://revistas.unal.edu.co/index.php/innovar/article/view/28707

Spiro, R., Feltovich, P., Jacobson, M., \& Coulson, R. (1999). Cognitive Flexibility, Constructivism, and Hypertext: Random Access Instruction for Advanced Knowledge Acquisition in III-Structured Domains. New Jersey, EE.UU.: Lawrence Erlbaum Associates, Inc., Publishers.

Tolman, E. (1932). Purpusive Behavior in Animals and Men. London, England: Century/Random House UK. 


\section{María Auxiliadora Pérez}

e-mail: maperez@ucla.edu.ve

Nacida en Barquisimeto, estado Lara, Venezuela. Soy

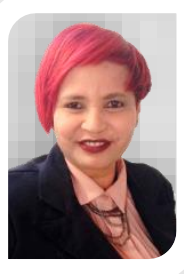
Ingeniero en Informática de profesión, egresada de la Universidad Centroccidental "Lisandro Alvarado", Magister en Ciencias de la Computación Mención Inteligencia Artificial de esta misma casa de estudios, Doctora en Gerencia Avanzada de la Universidad Fermín Toro, Cabudare, Venezuela. Actualmente me desempeño en el cargo de Profesor Asociado a Dedicación Exclusiva de la Universidad Centroccidental "Lisandro Alvarado", Docente de Postgrado en la Maestría en Ciencias de la Computación del DCyT-UCLA, Docente del Seminario de Innovación y Desarrollo del Doctorado en Ciencias Administrativas y Gerenciales del Decanato de Ciencias Económicas y Empresariales UCLA, Coordinadora de la Sección de Diseño e Innovación Educativa del Departamento Desarrollo Curricular UCLA, Coordinadora de la Comisión de Currículo del Programa de Ingeniería en Informática del DCyT-UCLA. En mi trayectoria profesional me destaco por haber sido Gerente de Telemática y Gerente Corporativo de Sistemas de empresas como: Organización Tunal, Proyectos e Instalaciones Electromecánicas C.A, Deel Ford C.A, Asesoría y Soluciones Empresariales en Sistemas, Carrocerías Larenses C.A., entre otras. Consultora de Innovación a cargo de Diseño y Desarrollo de Proyectos Tecnológicos Complejos e Innovadores de alta criticidad, para diversos clientes y mercados.

El contenido de este manuscrito se difunde bajo una Licencia de Creative Commons ReconocimientoNoComercial-Compartirlgual 4.0 Internacional 